\title{
Short term effects of corticosteroid pulse treatment on disease activity and the wellbeing of patients with active rheumatoid arthritis
}

\author{
J W G Jacobs, R Geenen, A W M Evers, C H M van Jaarsveld, F W Kraaimaat, \\ J W J Bijlsma
}

Department of Rheumatology and

Clinical Immunology, University Medical

Center, PO Box 85500,

3508 GA Utrecht, The

Netherlands

J W G Jacobs

C H M van Jaarsveld

J W J Bijlsma

Department of Health Psychology, Utrecht

University, The

Netherlands

$\mathrm{R}$ Geenen

Department of Medical Psychology,

University Hospital

Nijmegen, The

Netherlands

A W M Evers

F W Kraaimaat

Correspondence to: Dr Jacobs

g.dekruyf@digd.AZU.nl

Accepted for publication 15 May 2000

\begin{abstract}
Objective-To investigate the short term effects of corticosteroid pulse treatment (CPT) on disease activity, functional ability, and psychological wellbeing of patients with active rheumatoid arthritis (RA).

Methods-Of 66 consecutive patients with active RA admitted for CPT, erythrocyte sedimentation rate, $C$ reactive protein level, haemoglobin concentration, platelet count, duration of early morning stiffness, a joint score, and grip strength were assessed before and after CPT. Additionally, a health status questionnaire was administered. Effects of CPT were expressed as before to after intervention effect sizes and, to place them in perspective, compared with the (long term) effect sizes of disease modifying antirheumatic drug (DMARD) treatment in a historical contrast group of patients with early RA. Results-Statistically significant improvement from baseline in disease activity, physical functioning, and psychological wellbeing after CPT was seen, with moderate to large effect sizes, resembling the effects seen after DMARD treatment. Neither depression nor psychosis occurred during and after CPT.

Conclusion-Qualitatively and quantitatively the short term effects of CPT in patients with active established RA on various dimensions of health status resemble the long term effects of conventional DMARD treatment in patients with early RA. Psychological disorders do not seem to be common short term side effects of CPT in patients with active RA.

(Ann Rheum Dis 2001;60:61-64)
\end{abstract}

Recently published results of trials with low and high dose corticosteroid treatment (CT) are a reflection of renewed interest in this topic. ${ }^{12}$ Intravenous administration of high doses of corticosteroids (corticosteroid pulse therapy, CPT) is used in rheumatoid arthritis (RA) to suppress inflammation-for example, to bridge the lag time of recently prescribed disease modifying antirheumatic drugs (DMARDs), and to treat extra-articular complications such as vasculitis. ${ }^{3}$ With CPT the serious long term side effects of chronic oral corticosteroid treatment-with the exception of osteonecrosis - are avoided; short term side effect are usually mild. ${ }^{3}$ CPT may have less effect on bone resorption than continuous oral $\mathrm{CT}^{45}{ }^{5}$ The effect of CPT on clinical measures such as morning stiffness, pain, grip strength, and joint score varies in general between four and 10 weeks, but patients with long term remission of RA have been described. ${ }^{3}$

In various diseases the percentage of patients developing psychiatric symptoms (depression, hypomania, mania, or psychosis) during CT, especially given in high doses, has been reported to range from 3 to $75 \% .{ }^{6-8}$ In patients with RA, quantitative data on the effect of CPT or high dose oral CT on psychological wellbeing and quality of life are sparse. This study aimed at quantifying the short term effect of CPT on disease activity and self reported measures of psychosocial wellbeing and physical functioning of patients with active RA.

Patients and methods

Over a period of five years all consecutive patients with active RA, admitted to the rheumatology ward of the University Medical Center, Utrecht, to undergo CPT to bridge the lag time of a newly started DMARD, were included in this study after informed consent. If a patient was admitted more than once for CPT in this period of five years, only the first admission was used. None of the 66 patients receiving $\mathrm{CPT}$ (CPT group) refused to participate. Active disease was defined as the presence of at least two of the following three criteria: Thompson joint score $>10,{ }^{9}$ erythrocyte sedimentation rate (ESR) $\geqslant 28 \mathrm{~mm} / 1 \mathrm{st} \mathrm{h}$, and early morning stiffness $\geqslant 1$ hour. Each CPT regimen comprised three doses of $200 \mathrm{mg}$ dexamethasone (each dose 1000 mg methylprednisolone equivalent), given through an intravenous infusion on alternate days over a five day period. 
Table 1 Short term effects of corticoid pulse treatment (CPT) and long term effects of conventional disease modifying antirheumatic drug (DMARD) treatmentł. Results are given as means (SD) and effect sizes (ES)

\begin{tabular}{|c|c|c|c|c|c|c|}
\hline & \multicolumn{3}{|c|}{$C P T$ group $(n=66)$} & \multicolumn{3}{|c|}{ DMARD contrast group $(n=181)$} \\
\hline & Before & After & ES & $E S$ & Before & After \\
\hline $\mathrm{ESR}+(\mathrm{mm} / 1 \mathrm{st} \mathrm{h})$ & $68(33)$ & $38(27)$ & $1.01^{\star \star \star \star \star \star}$ & $0.60^{\star \star \star \star \star \star}$ & $41(28)$ & $26(24)$ \\
\hline $\mathrm{CRP}+(\mathrm{mg} / \mathrm{l})$ & $57(46)$ & $16(32)$ & $1.05^{\star \star \star \star \star \star}$ & $0.75^{\star \star \star \star \star \star}$ & $34(42)$ & $11(18)$ \\
\hline Haemoglobin $(\mathrm{mmol} / \mathrm{l})$ & $7.5(1.0)$ & $7.6(1.0)$ & 0.09 & $0.31^{\star \star \star \star \star}$ & $7.9(0.9)$ & $8.2(0.9)$ \\
\hline Platelets $\left(\times 10^{9} / 1\right)$ & $358(128)$ & $353(125)$ & 0.04 & $0.45^{\star \star \star \star \star \star}$ & $344(120)$ & $292(103)$ \\
\hline EMS† (min) & $126(110)$ & $34(43)$ & $1.17^{\star \star \star \star \star \star}$ & $0.54^{\star \star \star \star \star \star}$ & $111(153)$ & $44(94)$ \\
\hline Joint score† $(0-534)$ & $355(137)$ & $244(144)$ & $0.80^{\star \star \star \star \star \star}$ & $1.01^{\star \star \star \star \star \star}$ & $142(100)$ & $52(79)$ \\
\hline Grip strength $\dagger(\mathrm{kPa})$ & $8(11)$ & $15(14)$ & $0.56^{\star \star \star \star \star}$ & $0.40^{\star \star \star \star \star \star}$ & $30(22)$ & $40(25)$ \\
\hline \multicolumn{7}{|l|}{ IRGL scales: } \\
\hline Mobility (7-28) & $12.1(3.5)$ & $13.4(4.0)$ & $0.35^{\star}$ & $0.45^{\star \star \star \star \star \star}$ & $17.8(6.2)$ & $20.6(6.0)$ \\
\hline Self care $(8-32)$ & $14.9(5.2)$ & $17.8(5.5)$ & $0.55^{\star \star \star \star}$ & $0.51^{\star \star \star \star \star \star}$ & $22.6(6.2)$ & $25.7(6.1)$ \\
\hline Pain $(6-25)$ & $22.0(3.0)$ & $12.8(5.3)$ & $2.22^{\star \star \star \star \star}$ & $1.07^{\star \star \star \star \star}$ & $18.5(4.6)$ & $13.2(5.4)$ \\
\hline Depressed mood $(0-24)$ & $5.9(5.2)$ & $2.2(4.1)$ & $0.77^{\star \star \star \star \star \star}$ & $0.46^{\star \star \star \star \star \star}$ & $5.3(4.9)$ & $3.3(3.6)$ \\
\hline Cheerful mood (0-24) & $7.9(4.2)$ & $11.8(3.5)$ & $0.99^{\star \star \star \star \star \star}$ & $0.50^{\star \star \star \star \star \star}$ & $9.6(4.6)$ & $11.9(4.3)$ \\
\hline Anxiety $(10-40)$ & $21.9(5.0)$ & $19.2(4.9)$ & $0.54^{\star \star \star \star \star \star}$ & $0.38^{\star \star \star \star \star \star}$ & $20.4(6.3)$ & $18.1(5.9)$ \\
\hline Potential support $(5-20)$ & $16.2(4.1)$ & $15.8(4.2)$ & 0.10 & 0.11 & $16.8(3.6)$ & $16.4(3.7)$ \\
\hline Actual support (3-12) & $6.5(1.7)$ & $6.5(1.9)$ & 0.00 & 0.04 & $6.6(1.8)$ & $6.5(1.8)$ \\
\hline Impact on eating/sleeping $(2-8)$ & $4.3(1.3)$ & $3.8(1.2)$ & $0.44^{\star \star \star}$ & $0.46^{\star \star \star \star \star \star}$ & $4.2(1.6)$ & $3.5(1.5)$ \\
\hline Impact on relationships $(2-8)$ & $3.4(1.8)$ & $3.2(1.6)$ & 0.09 & 0.08 & $3.1(1.6)$ & $3.3(1.8)$ \\
\hline
\end{tabular}

†ESR = erythrocyte sedimentation rate, Westergren method, range $0-140$; CRP $=\mathrm{C}$ reactive protein, range $0-\infty$; EMS $=$ early morning stiffness; joint score $=$ according to Thompson; grip strength $=$ with Martin vigorimeter.

¥Second assessment (denoted "after" in the heading of the table) at 1.3 (SD 1.0) weeks for the CPT group and 12 months in the DMARD contrast group.

IStatistical significance levels of changes from baseline: ${ }^{\star} \mathrm{p}<0.05 ;{ }^{\star \star} \mathrm{p}<0.01 ;{ }^{\star \star \star} \mathrm{p}<0.001 ;{ }^{\star \star \star \star} \mathrm{p}<0.0001 ;{ }^{\star \star \star \star \star} \mathrm{p}<0.00001$; all statistically significant changes denote improvement.

At admission and 1-2 days before discharge from the hospital, the following variables were assessed: ESR, $C$ reactive protein level (CRP), haemoglobin concentration, platelet count, duration of early morning stiffness, the joint score according to Thompson, and grip strength (assessed in $\mathrm{kPa}$, Martin vigorimeter). In addition, the IRGL questionnaire (Impact of Rheumatic diseases on General health and Lifestyle) was administered. The IRGL is a health status questionnaire, developed from the Arthritis Impact Measurement Scales 1 (AIMS1), assessing physical, psychological, and social functioning as well as the impact of the disease on daily life. ${ }^{10}$ The scales of the IRGL differ in their individual ranges and are expressed in the original direction-for example, high values on the scale pain, and low values on the scales mobility and self care indicate a poor health status. Side effects, including psychosis or depression, were recorded by the doctor at clinical observation.

To put the (short term) effects and effect sizes of CPT in perspective we compared them with the (long term) effects and effect sizes of DMARD treatment in a historical contrast group of 181 outpatients with recent onset RA, evaluated at the start and after one year of treatment with a DMARD (starting with hydroxychloroquine, $400 \mathrm{mg} /$ day or aurothioglucose, $50 \mathrm{mg} /$ week or oral methotrexate, $7.5-15 \mathrm{mg} /$ week). ${ }^{11}$ The two groups cannot be compared directly, obviously, because the selection criteria and the periods between assessments differ. In the DMARD contrast group, the same variables as in the CPT group were assessed.

\section{STATISTICAL ANALYSIS}

Means and standard deviations (SD) of baseline scores, scores after treatment, and effect sizes (ES) of the variables were calculated. The larger the ES, the larger the effect; an ES of 0.20 denotes a small, of 0.50 a moderate, and of 0.80 a large effect. ${ }^{12}$ Within-group changes in the variables were tested for statistical significance with paired Student's $t$ tests where there was a normal distribution, and Wilcoxon signed rank tests for differences in medians where the distribution was not normal.

To investigate the number of patients with clinically relevant improvement on each variable in the CPT group, for each outcome variable the numbers of patients with deterioration, no clinically relevant change, or improvement from baseline were calculated. Deterioration was defined as a deterioration in a variable $>33 \%$ and no clinically relevant change as a change of $\leqslant 33 \%$; an improvement in a variable $>33 \%$ was defined as a clinically relevant improvement. ${ }^{13}$ Also, the numbers of patients with a clinically relevant overall response to treatment (individual patient's improvement) in the CPT and the DMARD contrast group were calculated. A clinically relevant overall response was defined as at least $20 \%$ improvement in joint score and at least $20 \%$ improvement in three of the four following end points: (a) ESR or CRP; (b) grip strength; (c) early morning stiffness; $(d)$ pain.

All tests were two sided; a $\mathrm{p}$ value $<0.05$ was considered to be significant. The statistical analyses were performed with the Number Cruncher Statistical system (NCSS) and SPSS/PC+ statistical packages (NCSS, Kaysville, Utah; SPSS Inc, Chicago, Illinois).

\section{Results}

The mean ages (SD) in the CPT group and DMARD contrast group were 58 (13) and 57 (14) years, respectively; duration of RA in the CPT group was 12 (10) years. The mean duration (SD) of admission for the CPT group was 1.3 (1.0) weeks and the median time period between assessments eight days; $66 \%$ of the patients in this group had used three or more DMARDs at the time of admission. 


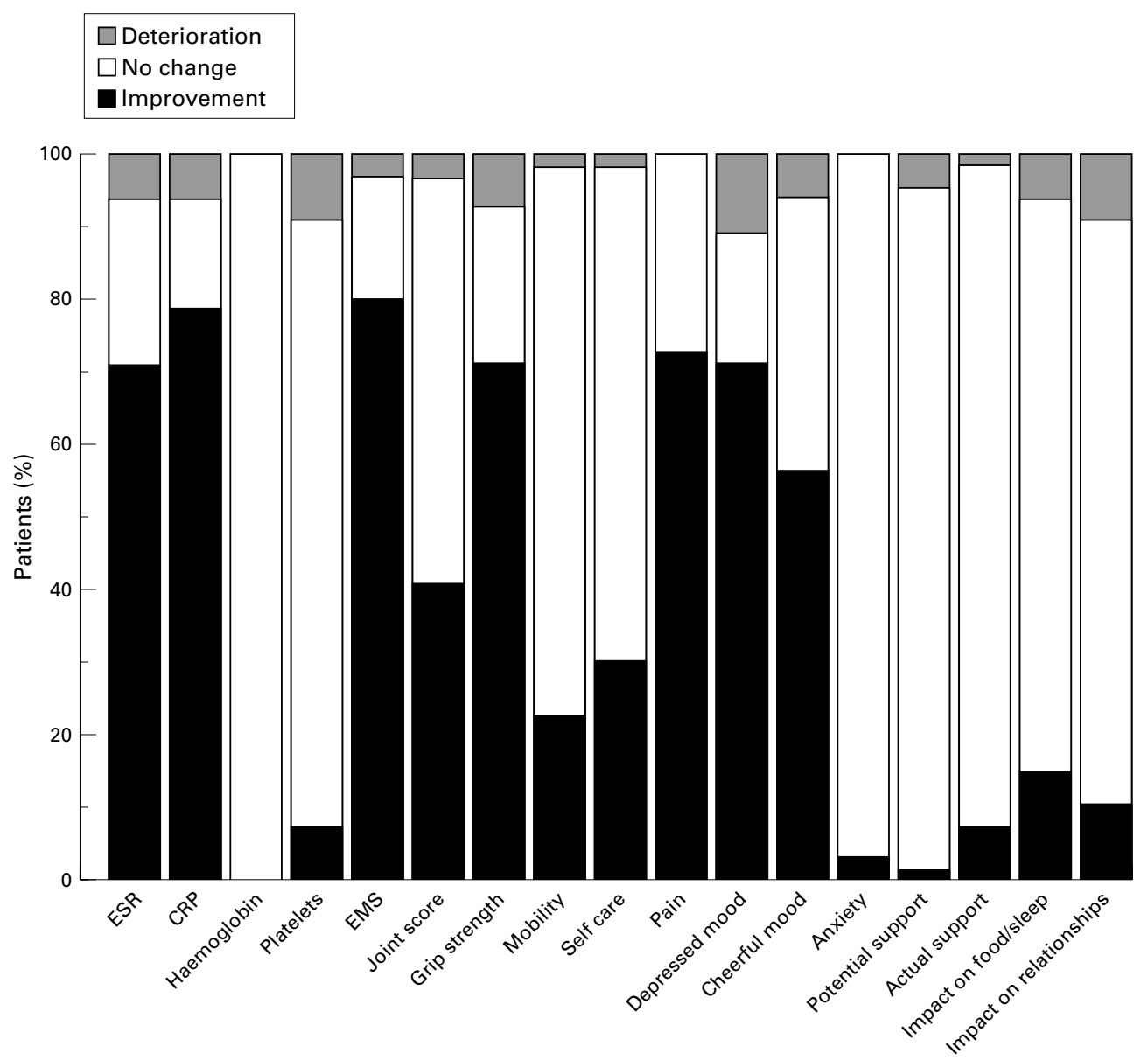

Figure 1 Percentages of patients with deterioration, no change, or improvement for each variable CPT group $(n=66)$, stacked bars. Improvement on platelets is a decrease in count.

Table 1 shows the means (SD) of the variable measures at the start and second assessment in both groups, together with the corresponding ES. Variables of disease activity and psychological wellbeing, in general, show a large ES and those reflecting functional ability a moderate ES. Figure 1 shows the percentages of patients in the CPT group with deterioration, no clinically relevant change, or improvement from baseline for each individual variable. More than $50 \%$ of patients improved by $33 \%$ or more in ESR, CRP, early morning stiffness, grip strength, pain, depressed mood, and cheerful mood. For the other variables, $>50 \%$ of the patients showed no clinically relevant change. In seven patients their depressed mood deteriorated; however, the mean score before treatment for these patients was 1 (range 0-3) and the mean score after treatment 4 (range 2-7) on a scale ranging from 0 (no depression) to 24 (severe depression). These deteriorations thus were small changes in the lower, nondepressive range of the scale. Clinical observation in the CPT group disclosed in many patients a sense of euphoria after treatment, but no cases of psychosis or depression. Common short term complications of CPT encountered were increase in blood pressure, hyperglycaemia, and facial flushing, as reported earlier. $^{3}$

In the CPT group the percentage of patients with a clinically relevant overall response to treatment (individual patient's improvement) was 53; in the DMARD contrast group the percentage was exactly the same.

\section{Discussion}

This study aimed at qualitatively and quantitatively analysing the short term effect of CPT on health status among patients with active RA; the results may be relevant also for treatment strategies with high dose oral CT. ${ }^{2}$ After CPT, clinically relevant changes are found for the same variables that show clinically relevant changes with DMARD treatment in three domains of health status: disease activity, physical functioning, and psychological wellbeing. In the CPT group the percentage of patients with a clinically relevant overall response to treatment was the same as in the DMARD contrast group.

In the CPT group, the IRGL showed enhanced psychological wellbeing, and clinical observation disclosed no depression or psychosis. So depression and psychosis do not seem to be common short term side effects of CPT in patients with active established RA. Psychological side effects generally begin during the first weeks after the start of treatment ${ }^{6}$; however, our results do not rule out the possibility of psychological disturbances later on. The incidence of depression and psychosis due to CPT in patients with active RA who improve within days may be different from the (high) 
incidence of psychological disorders in patients with other diseases-for example, in patients with nephritis, in whom no short term beneficial effects occur, or in whom short term negative effects of corticosteroids may even predominate, or in patients, in whom psychological disturbances may be part of the disease-for example, systemic lupus erythematosus.

1 Kirwan JR. Arthritis and Rheumatism Council Low-dose Glucocorticoid Study Group. The effect of glucocorticosteroids on joint destruction
Engl J Med 1995;333:142-6.

2 Boers M, Verhoeven AC, Markusse HM, van de Laar MJ, Westhovens R, Denderen JC, et al. Randomised comparison of combined step-down prednisolone, methotrexate and suphasalazine with suphasalazine alone in early rheumatoid arthritis. Lancet 1997;350:309-18.

3 Weusten BLAM, Jacobs JWG, Bijlsma JWJ. Corticosteroid pulse therapy in active rheumatoid arthritis. Semin Arthritis Rheum 1993;23:183-92.

4 Emkey RD, Lindsay R, Lyssy J, Weinberg JS, Dempster DW, Shen $\mathrm{V}$. The systemic effect of intraarticular administration of corticosteroid on markers of bone formation and bone resorption in patients with rheumatoid arthritis. Arthritis Rheum 1996;39:277-82.
5 Lems WF, Gerrits MI, Jacobs JWG, van Vugt RM, van Rijn HJM, Bijlsma JWJ. Changes in (markers of) bone metabolism during high dose corticosteroid pulse treatment in patients with rheumatoid arthritis. Ann Rheum Dis 1996;55:288-93.

6 Brown ES, Suppes T. Mood symptoms during corticosteroid therapy: a review. Harvard Review of Psychiatry 1998; 5:239-46.

7 Mitchell A, O'Keane V. Steroids and depression. Glucocorticoid steroids affect behaviour and mood. BMJ 1998;316:244.

8 Klein JF. Adverse psychiatric effects of systemic glucocorticoid therapy. Am Fam Physican 1992;46:1469-74

9 van der Brink HR, van der Heide A, Jacobs JWG, van der Veen MJ, Bijlsma JWJ. Evaluation of the Thompson articular index. J Rheumatol 1993;20:28-32.

10 Evers AWM, Taal E, Kraaimaat FW, Jacobs JWG, Abdel-Nasser A, Rasker JJ, et al. A comparison of two recently developed health status instruments for patients with arthritis: Dutch-AIMS2 and IRGL. Br J Rheumatol 1998;37:157-64.

11 van der Heide A, Jacobs JWG, Bijlsma JWJ, Heurkens AHM, van Booma-Frankfort C, van der Veen MJ, et al. The effectiveness of early treatment with "second-line" antirheumatic drugs. Ann Intern Med 1996;124:699-707.

12 Cohen I. Statistical power analysis for the behavioral sciences. New York: Academic Press, 1977.

13 Goldsmith CH, Boers M, Bombardier C, Tugwell P. Criteria for clinically important changes in outcomes: development, scoring and evaluation of rheumatoid arthritis patients and trial profiles. J Rheumatol 1993;20:561-5. 